\title{
Intervention to reduce C-reactive protein determination requests for acute infections at an emergency department
}

\author{
I S Santos, ${ }^{1}$ I M Benseñor, ${ }^{1,2}$ J B A Machado, ${ }^{3}$ L M G Fedeli, ${ }^{3}$ P A Lotufo ${ }^{1,2}$
}

${ }^{1}$ University of São Paulo Medical School, Internal Medicine Department, São Paulo, Brazil ${ }^{2}$ University Hospital of the University of São Paulo, Internal Medicine Division, São Paulo, Brazil

${ }^{3}$ University Hospital of the University of São Paulo, Central Laboratory, São Paulo, Brazil

\section{Correspondence to}

Dr Itamar de Souza Santos, Hospital Universitario-USP, 2565 Professor Lineu Prestes Avenue 30 andar, Centro de Pesquisa Clínica, São Paulo, Brazil; itamarss@usp.br

Accepted 22 December 2011

Published Online First 3 February 2012

\section{ABSTRACT}

Introduction C-reactive protein (CRP) levels rise during inflammatory processes and have been ordered for rheumatic disease follow-up since the 1950s. The number of tests ordered in the emergency setting has increased, but without evident improvement in medical care quality.

Objective To determine the pattern of CRP determinations in the emergency department (ED) of a university hospital in Sao Paulo, Brazil, and to evaluate the effect of an intervention with staff and students about the best use of the test in the ED.

Methods Data regarding CRP testing requests, related diagnoses and the number of monthly consultations in the hospital ED were analysed before and after the intervention. Because of an increase in CRP measurement requests from 2007 to 2009, the author started discussing the role of CRP determinations in the medical decision-making process in early 2010. Staff and faculty members openly discussed the pattern of requests in the hospital and related current medical literature. During 2010, the medical staff worked as multipliers to change the behaviour of new students and residents. The results of the first 4 months after the intervention were presented at another general meeting in July 2010.

Results From 2007 to 2009, there were 11786 CRP measurement requests with a clear exponential trend. After the intervention, during the calendar year 2010, there was a $48 \%$ reduction in adjusted annual CRP requests. Pneumonia, fever and urinary tract infections were the most common reasons for CRP requests. Discussion Inexpensive, well-directed, interactive educational interventions may affect professional behaviour and curb rates of laboratory tests.

Unnecessary testing may be harmful and is associated with high costs and wasted resources. Efforts to minimise this situation have been described in the medical literature. Solomon et $a l^{1}{ }^{1}$ in a systematic review of 49 studies focusing on single or multiple diagnostic tests, concluded that interventions targeting consensual behavioural changes may be effective. Later, Stuart et $a l^{2}$ proposed a protocol for ordering tests in the emergency department (ED) that, along with an education programme for medical staff and a feedback process, resulted in a $40 \%$ decrease in orders for all diagnostic tests in the ED of a teaching hospital.

C-reactive protein (CRP) is a well-recognised acute-phase protein that generally increases markedly and quickly in the setting of an inflammatory event. $^{3}$ Infections, auto-immunity, malignancies, trauma and other processes may initiate this response. ${ }^{4}$ CRP determination has a potential role in patients with acute infections. Some of these studies are set in intensive care units (ICU). ${ }^{5}{ }^{6}$ In the ED of an urban, university-based medical centre, Lee et $a l^{7}$ evaluated the prognostic value of CRP and procalcitonin in patients with sepsis They found that a score based on medical history, clinical examination, blood cell counts and a chest x-ray when necessary (all usually done in such a setting) was better at predicting mortality than both CRP and procalcitonin, suggesting that these examinationss would add little to clinical judgement. ${ }^{8}$ In a convenience sample of individuals who underwent the test, Adams ${ }^{9}$ reported that CRP had little diagnostic utility for identifying bacteraemic patients in the ED. Similarly, in individuals with an acute exacerbation of chronic obstructive pulmonary disease, CRP levels were not associated with either short or long-term outcomes. ${ }^{10} 11$

Data on how CRP findings may affect medical action (eg, prescribing antibiotics and/or discharging patients home) in real-life emergency settings are even scarcer. Flanders et al ${ }^{12}$ found higher CRP levels in patients with an acute cough caused by pneumonia, but could not determine a clear cut-off level, and the area under the receiver operating characteristic curve was smaller than the clinical prediction rule set by Heckerling et al. ${ }^{13} \mathrm{~A}$ systematic review by van der Meer et al ${ }^{14}$ concluded that CRP determinations could not identify an infectious aetiology for a pulmonary infiltrate in a chest x-ray. Moreover, CRP levels could not be adequately associated with a bacterial aetiology for infectious infiltrates. These findings led Au-Jong and Coats, ${ }^{15}$ in a recent revision, to state that CRP levels should not be used to differentiate between heart failure and pneumonia.

The current study was designed to identify the pattern of CRP test requests in the ED of a university hospital in Sao Paulo, Brazil, in recent years and identify the benefits of a thorough discussion that occurred in early 2010 with the staff and students about the best use of the test in an ED.

\section{METHODS \\ Setting}

The Hospital Universitario of the University of São Paulo (HU-USP) is a medium-sized (260-bed) public teaching hospital in Sao Paulo, Brazil. It comprises ED and inpatient (ICU and non-ICU) and ambulatory units. HU-USP is organised into four main divisions (internal medicine, general surgery, obstetrics and gynaecology, and paediatrics) 
and is a training clinical hospital for approximately 360 undergraduate students and more than 200 residents in different medical specialities every year. The HU-USP ED has separate sectors for child and adult care. Patients may present spontaneously for a consultation or be referred by primary care units located in the hospital's catchment area of 425000 inhabitants. Laboratory examinations and, in particular, CRP testing are performed at the clinicians' discretion, with no mandatory protocol or restrictive interventions. The entire hospital uses a computer system to manage the requests, completions and results of laboratory tests. This system is accessed using a personal password. Access to system features varies across different healthcare professional categories; medical students in clerkship, residents and hospital medical staff can request laboratory tests.

\section{Procedures}

We obtained data regarding CRP measurement requests for patients aged 15 years and older in the hospital ED from January 2007 to December 2010. For these requests, we obtained data on the assay technique, defined as high-sensitivity or standard technique. For both techniques, the CRP determination was performed using the BN Systems (Siemens) immunonephelometry method. The techniques differ in the initial sample dilution; 1:400 for standard CRP and 1:20 for high-sensitivity CRP. We also retrieved information about the date of the request, requester's name, and the diagnosis associated with the consultation that led to the request, which was classified according to the 10th edition of the International Classification of Diseases (ICD-10). ${ }^{16}$

The request dates were stratified by month and year. Requester name and request date were used to determine professional category (medical students, residents, medical staff, or other/undefined) based on HU-USP and University of São Paulo Medical School official records.

\section{Intervention}

Aware of a remarkable increase in CRP test requests from 2007 to 2009, members of the HU-USP internal medicine division, including faculty members, medical staff, residents and students, started discussing the role of CRP determinations in the decision-making process in early 2010. Before a general meeting scheduled early in 2010, a thorough revision of CRP test requests in the hospital was performed. Although the number of requests had increased in almost all settings of the internal medicine division, it was largely due to an increase in requests originating from the ED.

In January 2010, these data were presented and discussed at a general meeting specifically focused on the role of the examination for outpatient and non-ICU inpatient care. Residents, interns and internal medicine division staff attended this general meeting. This included not only those who worked in the ED, but also those involved in inpatient care (where the number of $\mathrm{CRP}$ test requests had also increased over the past few years, data not shown), the ambulatory unit and ICU. The meeting was previously scheduled and, although not obligatory, those not on duty on the day of the discussion were also encouraged to participate. A review of the related medical literature and test performance in various situations (sensitivity, specificity and likelihood ratios) was presented, as well as related tangible and intangible costs. An open discussion then followed to identify patterns of request and suitability. There was broad consensus that the test was over-solicited.

It is important to note that, at a teaching hospital like ours, residents and students typically change every 1 or 2 months because they rotate to different institutions during the year. At our institution, it is characteristic that, while students and residents rotate through the emergency department, there is a close relationship between them and the medical staff. Given this relationship, we decided that the medical staff should act as multipliers. We concluded that this would be the best way to provide for further discussion and change the behaviour of new groups. Also, to strengthen this new phase of the intervention and reinforce information regarding the intervention's potential, the results of the first 4 months after the intervention were presented at another general meeting in July 2010. During the intervention period, there were no mandatory, restrictive, or punitive strategies. Individual medical decisions continued to determine which patients received CRP testing. This allowed the impact of this open, evidence-based discussion, focused on this selected point of medical action, to be measured.

\section{Statistical analysis}

Descriptive statistics for the number of ED consultations and CRP test requests were performed using SPSS 16.0. In addition, we adjusted the annual CRP test requests to the number of ED consultations, using the ratio of CRP test requests per $1000 \mathrm{ED}$ consultations to make the data comparable. We also calculated the percentage of CRP test requests in the hospital generated in the ED by month and by year. We compared the number of requests for each professional category from 2007 to 2010. ICD10 classifications associated with CRP test requests were analysed both as specific disorders and ICD chapters as defined by the WHO.

We applied the joinpoint regression program 3.5.1 (Statistical Research and Applications Branch, National Cancer Institute), a log-linear model using Poisson regression, to identify trends in the ratio of CRP test requests per $1000 \mathrm{ED}$ consultations during the 48-month observation period. We used a minimum of three observations from each end of the data range, and four observations between each joinpoint to identify a trend change. Because our observation used monthly intervals, the monthly percentage change (MPC) was calculated for each modelled segment. The significance level was set at $\alpha=0.05$.

\section{RESULTS}

From January 2007 to December 2010, there were 11786 requests for CRP determinations in the $\mathrm{ED}$. Of these, 11689 (99.2\%) were standard CRP requests. Table 1 shows the number of CRP test requests for each year. The number of ED consultations decreased slightly during this period. From 2007 to 2009, both the ratio of CRP test requests per $1000 \mathrm{ED}$ consultations and the rate of CRP test requests generated in the $\mathrm{ED}$ rose markedly.

Table 1 Change in the pattern of CRP test requests in the $E D$, number of ED consultations, ratio of CRP test requests per $1000 \mathrm{ED}$ consultations and rate of hospital CRP test requests generated in the ED from 2007 to 2010

\begin{tabular}{|c|c|c|c|c|}
\hline & \multicolumn{4}{|l|}{ Year } \\
\hline & 2007 & 2008 & 2009 & 2010 \\
\hline CRP requests in ED & 1073 & 2233 & 5602 & 2878 \\
\hline ED consultations & 212127 & 208394 & 194951 & 194338 \\
\hline $\begin{array}{l}\text { Ratio of CRP test requests } \\
\text { per } 1000 \text { ED consultations }\end{array}$ & 5.1 & 10.7 & 28.7 & 14.8 \\
\hline $\begin{array}{l}\text { Percentage of hospital CRP test } \\
\text { requests generated in the ED }\end{array}$ & $24.8 \%$ & $25.5 \%$ & $38.6 \%$ & $26.4 \%$ \\
\hline
\end{tabular}

CRP, C-reactive protein; ED, emergency department. 
Table 2 CRP test requests according to each professional category in the ED by the year of the request

\begin{tabular}{lrrrrr}
\hline & Year & & & & \\
\cline { 2 - 5 } Professional category & $\mathbf{2 0 0 7}$ & $\mathbf{2 0 0 8}$ & $\mathbf{2 0 0 9}$ & $\mathbf{2 0 1 0}$ & Total \\
\hline Medical students & 209 & 532 & 1502 & 582 & 2825 \\
Medical residents & 537 & 878 & 2032 & 1221 & 4668 \\
Medical staff & 316 & 803 & 2002 & 1050 & 4171 \\
Other/unidentified & 11 & 20 & 66 & 25 & 122 \\
Total & 1073 & 2233 & 5602 & 2878 & 11786 \\
\hline
\end{tabular}

CRP, C-reactive protein; ED, emergency department.

Table 2 shows the number of requests according to each professional category. In all categories, there was a clear increase in requests. In 2010, however, there was a 48\% reduction in the ratio of CRP test requests per $1000 \mathrm{ED}$ consultations. Figure 1 details the request pattern during the observation period. A significant increase in the ratio of CRP test requests per $1000 \mathrm{ED}$ consultations occurred from January to November 2007 (MPC for segment $1=24.1 \%$; $95 \%$ CI $11.5 \%$ to $38.0 \%$ ) and from April 2008 to November 2009 (MPC for segment $3=10.1 \%$; $95 \%$ CI $8.3 \%$ to $11.9 \%$ ). A significant sharp decline was observed from December 2009 to April 2010 (MPC for segment 4=-24.2\%; $95 \%$ CI $-34.6 \%$ to $-12.2 \%)$. An inflection point followed by non-significant trends was observed from December 2007 to March 2008 (MPC for segment 2=-12.7\%; 95\% CI $-41.2 \%$ to $+29.7 \%$ ) and from May 2010 to December 2010 (MPC for segment $5=3.7 \%$; $95 \% \mathrm{CI}-3.2 \%$ to $+11.1 \%$ ). The annual reduction observed after the intervention was consistent in all professional subgroups, but was even more intense among undergraduates $(61.3 \%$ for medical students, $39.9 \%$ for medical residents and $47.6 \%$ for medical staff; $\mathrm{p}<0.001)$.

For $8881(75.4 \%)$ of the CRP test requests in the ED, it was possible to access the medical discharge diagnoses. Because some visits or hospitalisations may have more than one diagnosis, 12418 discharge diagnoses were registered. Table 3 shows the 10 most frequent specific diagnoses related to requests for CRP determination. The most frequent diagnoses were the same throughout the observation period, and the main diagnosis did not change after the intervention.

\section{DISCUSSION}

It is widely recognised that unnecessary testing in the ED is associated with higher tangible and intangible costs. This increase may have repercussions in terms of material use, staff work, needless further testing, and time spent by the patient; therefore, initiatives to limit this hazardous practice are desirable.

Our findings uncovered a potentially troublesome scenario. The medical literature does not currently support the use of CRP testing in decision-making processes in the ED. Moreover, ED patients, unlike some patients in the ICU setting, can usually provide valuable information about symptoms. Their signs of infection may be much easier to identify. It is reasonable to think that non-specific information such as CRP levels may benefit doctors in the ICU, but not those working in the ED. Because of this, in our opinion, academic findings in one setting may not be transposed to the other.

Data for this study were obtained from a university hospital, where almost 600 medical students and residents are trained each year. In a setting such as this, interventions to improve the decision-making process in patient management are very important. In January 2010, an evidence-based, thorough discussion with hospital staff, residents, students and faculty members about the real need for CRP testing in the ED started during a general meeting. That meeting was followed by close contact discussions, provided by the medical staff, with the new groups of students and residents during the year. This strategy led to a remarkable decrease in the number of requests. It was expected that the reduction in the number of requests would occur mainly during the first months after beginning the intervention, as observed in the results section. This period, characterised by a decline in requests for CRP testing, was followed by a stabilisation period. The very discrete increase ( $<4 \%$ monthly) observed during this phase was non-significant, showing a constant number of requests for CRP tests. This contrasts with the slope of the increase observed during the same periods in the years before the intervention (2007-9). It is now also our policy to strengthen and expand discussions like this one to other situations in patient care. Although it is not possible to determine exactly to what extent the drop in the number of requests in 2010 was due to this discussion, clearly an upward trend was reversed. Also worth noting is that this effective strategy was based on behaviour changes and did not include any barriers or limitations to test requests. Based on our data, medical students may be especially responsive to such interventions.

On the other hand, other authors have studied new potential roles for CRP testing in the ED. Paran et $a l^{17}$ found that the CRP
Figure 1 Modelled trends for the number of $\mathrm{C}$-reactive protein (CRP) test requests per 1000 emergency department (ED) consultations, from month 1 (January 2007) to 48 (December 2010). Dots represent observed values. ${ }^{*} \mathrm{p}<0.05$ for MPC in segments 1, 3 and 4 . MPC, monthly percentage change.

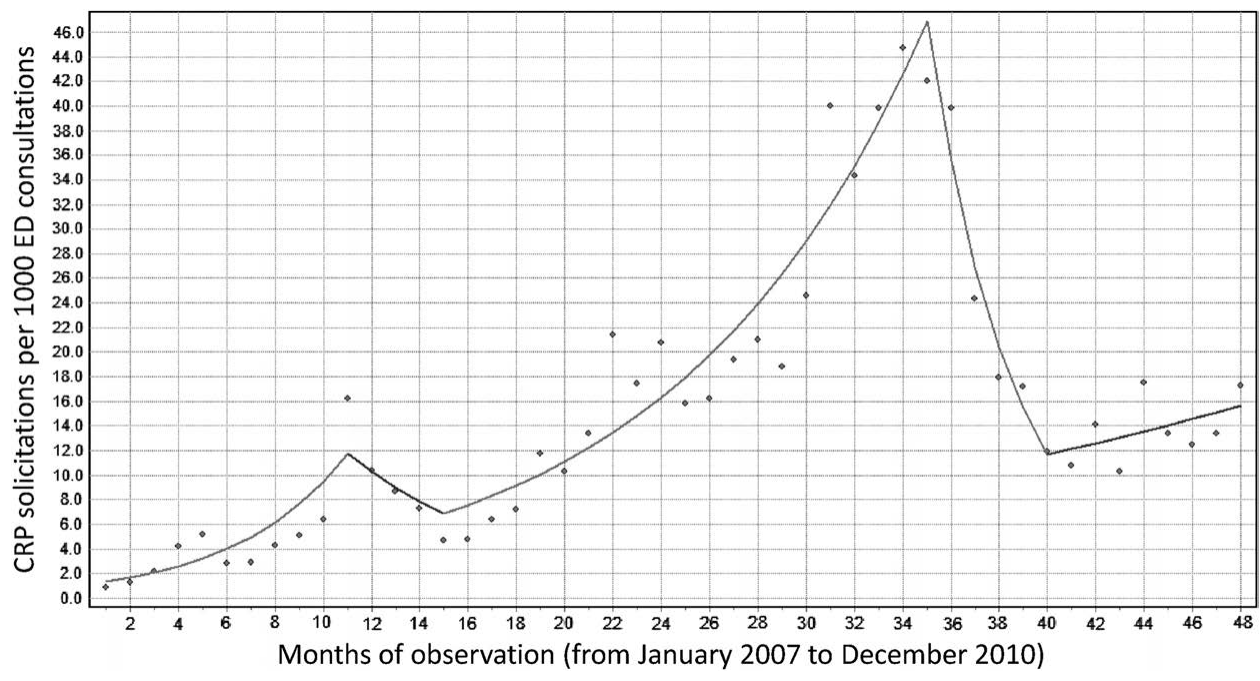


Table 3 The 10 most frequent diagnoses associated with CRP test requests in the $\mathrm{ED}$

\begin{tabular}{lll}
\hline ICD-10 code & Description & n \\
\hline J18 & Pneumonia & 885 \\
R50 & Fever & 475 \\
N39 & Disorders of the urinary system, including & 386 \\
& urinary tract infection & \\
R10 & Abdominal and pelvic pain & 315 \\
J44 & Chronic obstructive pulmonary disease & 308 \\
I50 & Heart failure & 301 \\
A09 & Diarrhoea and gastroenteritis & 265 \\
J98 & Other respiratory disorders & 202 \\
042 & Premature rupture of membranes & 199 \\
R51 & Headache & 182 \\
\hline
\end{tabular}

CRP, C-reactive protein; ED, emergency department; ICD-10, International Classification of Diseases, version 10.

velocity (calculated as the CRP level at admission divided by the duration of fever in hours) had greater accuracy than the CRP level or the duration of fever alone for detecting bacterial aetiology in patients with fever. However, although this finding potentially represents a step forward in defining a role for CRP determinations in the $\mathrm{ED}$, it is our understanding that to make this new strategy a reality, studies should address the extent to which it actually enhances medical decision-making and, moreover, patient outcome. Such studies should also include cost-benefit analyses.

Our study has a few strengths. It was possible to determine the diagnosis that originated the medical consultation for the majority of patients. Furthermore, the number of CRP test requests for which it was not possible to determine the requester was very small. However, this study design could not assess the number of cases in which the CRP determination influenced medical decisions and the outcomes of the decisions. Data on consultations with individuals for whom the examination was not performed were unavailable, so it was not possible to determine the ratio of CRP test requests per $1000 \mathrm{ED}$ consultations for each professional category.

In conclusion, the medical staff and managers involved in clinical practice, especially at academic centres, must be aware of the harm caused by unnecessary testing. Identifying specific problems and establishing intervention strategies may be of great use for staff, patients and students.

Competing interests The authors have no competing interests relating to the submitted work. Outside the present work, PAL has acted as a consultant for Astra-Zeneca and has received payment for manuscript preparation from Bristol
Myers Squibb. IMB is responsible for grants (for the institution) from FAPESP and FINEP (government granting agencies), and PAL is responsible for grants (for the institution) from Eli-Lilly, Daichi and Novartis.

Ethics approval The study was registered and approved by the Internal Review Board of the Hospital Universitario da Universidade de Sao Paulo, Sao Paulo, Brazil.

Contributors ISS collaborated in the study design, data collection, statistical analysis and wrote the paper. IMB and PAL collaborated in the study design and wrote the paper. JBAM and LMGF collaborated in the study design and assisted with writing the article.

Provenance and peer review Not commissioned; externally peer reviewed.

\section{REFERENCES}

1. Solomon DH, Hashimoto H, Daltroy L, et al. Techniques to improve physicians' use of diagnostic tests. JAMA 1998;280:2020-7.

2. Stuart PJ, Crooks S, Proton M. An interventional program for diagnostic testing in the emergency department. Med J Aust 2002;177:131-4.

3. Gabay C, Kushner I. Acute-phase proteins and other systemic responses to inflamattion. N Engl J Med 1999:340:448-54.

4. Ablij HC, Meinders AE. C-reactive protein: history and revival. Eur J Intern Med 2002:13:412-22.

5. Povoa P, Coelho L, Almeida E, et al. Early identification of intensive care unitacquired infections with daily monitoring of $\mathrm{C}$-reactive protein: a prospective observational study. Crit Care 2006;10:R63.

6. Fraunberger $\mathbf{P}$, Wang $Y$, Holler $E$, et al. Prognostic value of interleukin-6, procalcitonin and $\mathrm{C}$-reactive protein levels in intensive care unit patients during firs increase of fever. Shock 2006;26:10-12.

7. Lee CC, Chen SY, Tsai CL, et al. Prognostic value of mortality in emergency department sepsis score, procalcitonin, and C-reactive protein in patients with sepsis at the emergency department. Shock 2008;29:322-7.

8. Carpenter CR, Keim SM, Upadhye S, et al. Risk stratification of the potentially septic patient in the emergency department: the mortality in the emergency department sepsis (MEDS) score. J Emerg Med 2009;37:319-27.

9. Adams NG. Diagnostic use of C-reactive protein in bacteraemic emergency department patients. Emerg Med Australas 2005;17:371-5.

10. Antonescu_Turcu AL, Tomic R. C-reactive protein and copeptin: prognostic predictors in chronic obstructive pulmonary disease exacerbations. Curr Opin Pulmon Med 2009;15:120-5

11. Stolz D, Christ-Crain M, Morgenthaler NG, et al. Copeptin, C-reactive protein and procalcitonin as prognostic biomarkers in acute exacerbation of COPD. Chest 2007:131:1058-67.

12. Flanders SA, Stein J, Shochat G, et al. Performance of a bedside C-reactive protein test in the diagnosis of community-acquired pneumonia in adults with acute cough Am J Med 2004;116:529-35.

13. Heckerling PS, Tape TG, Wigton RS, et al. Clinical prediction rule for pulmonary infiltrates. Ann Intern Med 1990:113:664-70.

14. Van der Meer V, Neven AK, van den Broek PJ, et al. Diagnostic value of $C$ reactive protein in infections of the lower respiratory tract: systematic review. BMJ 2005;331:26.

15. Au-Jong A, Coats T. BET2: C-reactive protein in the differential diagnosis of heart failure and chest infection. Emerg Med J 2009;26:58-9.

16. World Health Organization. International Classification of Diseases, 10th edn. http://apps.who.int/classifications/apps/icd/icd10online (accessed 7 Jul 2011).

17. Paran Y, Yablecovitch D, Choshen G, et al. C-reactive protein velocity to distinguish febrile bacterial infections from non-bacterial febrile illnesses in the emergency department. Crit Care 2009;13:R50. 